\title{
Short-time traffic flow prediction based on immune particle swarm neural networks
}

\author{
Xiaomo Yu \\ N/A \\ Yuheng Kang \\ N/A \\ Zhou Shen ( $\sim$ shenzhou.research@gmail.com ) \\ N/A
}

\section{Research Article}

Keywords: Short-time traffic flow prediction

Posted Date: August 3rd, 2021

DOI: https://doi.org/10.21203/rs.3.rs-778025/v1

License: (1) This work is licensed under a Creative Commons Attribution 4.0 International License.

Read Full License 


\title{
Short-time traffic flow prediction based on immune particle swarm neural networks
}

\author{
Xiaomo Yu, Yuheng Kang, and Zhou Shen*
}

\begin{abstract}
The concentration-based selection mechanism in the immune theory can avoid the shortcomings of the particle swarm algorithm in balancing population convergence and individual diversity, and enable the improved particle swarm algorithm to optimize the configuration of BP neural network parameters and improve the accuracy of short-term traffic flow prediction. The simulation experiments show that the immune particle swarm optimized BP neural network can effectively improve the prediction accuracy of short-term traffic flow and reduce the prediction error.
\end{abstract}

\section{Introduction}

Short-time traffic flow prediction is to use the historical data of traffic flow to predict the future road traffic flow in real time (usually within 15 minutes) [1], to provide design basis and decision support for traffic signal control and dynamic route guidance $[2-5]$. However, the factors affecting the efficiency of urban roads are highly dynamic, non-linear, uncertain, periodic, non-stationary and spatially dependent, and it is difficult to give precise analytical expressions [6-9]. Artificial neural networks have the characteristics of non-linear description, parallel distributed processing, learning and self-adaptation, good at dealing with multivariate systems and with a certain degree of fault tolerance, which are more suitable for traffic flow prediction $[10,11]$. The particle swarm algorithm relies on the unique memory among the population particles and can dynamically track the current search situation to adjust the search strategy, which can avoid the blindness of evolutionary operation to a certain extent.

The bio-immune property can achieve a better balance between convergence and individual diversity in the evolutionary process of particle swarm, solve the problem of slow convergence and easy to fall into local optimum due to the gradual convergence of particles in the later stage of the algorithm, and improve the global search ability of the algorithm. The immune particle swarm algorithm is used to train the structure, initial weights and thresholds of the BP neural network to overcome the inherent defects of the BP neural network, such as slow convergence speed and the tendency to fall into local optimality. The simulation

\footnotetext{
${ }^{*}$ The authors declare no competing interests.
} 
results show that the trained $\mathrm{BP}$ neural network has faster convergence speed compared with the normal particle swarm algorithm under the same accuracy and error requirements.

\section{BP neural network model}

As a multilayer feed-forward neural network, the neurons of BP neural network have $\mathrm{n}$ inputs, each input is interconnected with the next layer by an appropriate weight $w$. The network output can be expressed as

$$
y=f(w \times p, b)
$$

BP neural networks usually have one or more hidden layers, and the transfer function $f$ of neurons in the layers usually uses a Sigmoid-type transform function (such as tangent-type function tansig or logsig function) to map the input quantity to a continuous output quantity between -1 and 1, to achieve any nonlinear mapping from input to output. The neuron transfer function $\mathrm{f}$ in the output layer is generally a purely linear purelin transform function [12-15].

After determining the structure of the BP neural network, the network is trained using the input and output sample sets, and the learning algorithm of error back propagation is used to adjust the weights wi and thresholds $\theta \mathrm{i}$ in the network to achieve the given input-output mapping relationship.

\section{Particle swarm algorithms}

\subsection{Basic particle swarm algorithm}

The particle swarm algorithm uses the "velocity-displacement" model to solve the optimization problem. In a swarm of particles of dimension $\mathrm{m}$, the position of particle $\mathrm{i}$ in d-dimensional space is represented by $\mathrm{Xi}=(\mathrm{xi} 1, \mathrm{xi} 2, \ldots, \mathrm{xid})$ and its velocity is represented by $\mathrm{Vi}=($ vi 1, vi $2, \ldots, \mathrm{vid})$, where $\mathrm{i}=1,2, \ldots, \mathrm{m}$. In addition, the velocity is represented by $\mathrm{Pi}=($ pi1,pi2,..,pid $)$ and $\mathrm{Pg}=(\operatorname{pg} 1, \mathrm{pg} 2, \ldots, \mathrm{pid})$. pi1,pi2,..,pid) and $\mathrm{Pg}=(\mathrm{pg} 1, \mathrm{pg} 2, \ldots, \mathrm{pgd})$ denote the optimal position currently searched for by particle $i$ and the whole population, respectively. Evaluate the position information of particle $\mathrm{i}$ in the kth generation according to the objective function, calculate the optimal position $\mathrm{Pi}$ of this particle and the optimal position $\mathrm{Pg}$ of the population, and generate the velocity and position information of the next generation of particles according to the following equations:

$$
\begin{gathered}
v_{i d}^{k+1}=w \times v_{i d}^{k}+c_{1} \times \eta_{1} \times\left(p_{i d}-x_{i d}^{k}\right)+ \\
c_{2} \times \eta_{2} \times\left(p_{g d}-x_{i d}^{k}\right), \\
x_{i d}^{k+1}=x_{i d}^{k}+v_{i d}^{k+1} .
\end{gathered}
$$


where $\mathrm{w}$ is the inertia weight, which can be updated according to equation

$$
w=w_{\max }-\text { Iter } \times \frac{\left(w_{\max }-w_{\min }\right)}{\text { Iter } \max }
$$

based on the evolutionary algebra Iter. $\mathrm{c} 1, \mathrm{c} 2$ are acceleration coefficients, which are used to regulate the maximum step of flight in the direction of the individual best position versus the global best position. $\eta_{1}$ and $\eta_{2}$ are random numbers uniformly distributed in the interval $[0,1]$.

Each particle searches and tracks individual and global extrema in the solution space until a specified maximum number of iterations or minimum error accuracy is reached.

\subsection{Particle swarm algorithms based on immunological memory and immunomodulation}

According to the characteristics of the biological immune system, in the immune algorithm, the antigen is considered as the optimal solution to the problem, and the antibody produced by immunisation is considered as the candidate solution to the problem. The degree of similarity between antibody and antigen or between antibodies is expressed as a fitness function to establish an evaluation mechanism between antibody and antigen.

The immune algorithm uses a diversity maintenance strategy based on a concentration selection mechanism to maintain a certain concentration of particles at each fitness level in the particle population, in order to prevent the algorithm from falling into a local optimum. The concentration of particle xi is defined as follows

$$
\begin{gathered}
D\left(x_{i}\right)=\frac{1}{\sum_{j=1}^{n+m}\left|f\left(x_{i}\right)-f\left(x_{j}\right)\right|} \\
i=1,2, \cdots, n+m .
\end{gathered}
$$

Selection probability formula based on particle concentration

$$
\begin{aligned}
& P\left(x_{i}\right)=\frac{\frac{1}{D\left(x_{i}\right)}}{\sum_{i=1}^{n+m} \frac{1}{D\left(x_{i}\right)}}=\frac{\sum_{j=1}^{n+m}\left|f\left(x_{i}\right)-f\left(x_{j}\right)\right|}{\sum_{i=1}^{n+m} \sum_{j=1}^{n+m}\left|f\left(x_{i}\right)-f\left(x_{j}\right)\right|} \\
& \mathrm{i}=1,2, \cdots, \mathrm{n}+\mathrm{m}
\end{aligned}
$$

where $f(x i)$ denotes the value of the fitness function of particle xi.

The probability of particle i being selected is inversely proportional to its concentration, so that particles with low adaptive values can also have a chance to evolve, in order to overcome the shortcomings of the particle swarm algorithm in the later stages of the "convergence" of particles, resulting in poor search efficiency in the local solution space, and improve the global search ability of the population. The flow of the algorithm is as follows: 
Step 1: Initialize the particle swarm. According to the basic particle swarm algorithm, the initial particle swarm Pop0 with population size $\mathrm{n}$ is randomly generated, and the initial position $\mathrm{Xi} 0$ and velocity $\mathrm{Vi} 0$ of the particles are set, $\mathrm{i}=1 \ldots \mathrm{n}$.

Step 2: Calculate the individual adaptation value of the particle, determine the individual extreme value $\mathrm{Pi}$ and the global extreme value $\mathrm{Pg}$ of the current particle, and set Pg also as the immune memory particle and store it in the memory bank.

Step 3: Generate new particles. Firstly, the positions and velocities of the $\mathrm{n}$ particles of the previous generation are updated according to Eqs. (2) and (3), and then $m$ new particles are generated randomly in the population.

Step 4: Concentration-based particle selection. The selection probability of the newly generated $n+m$ particles is calculated according to equation (5) and equation (6), and the $\mathrm{n}$ particles with higher probability are selected to form the kth generation particle population Popk.

Step 5: Particle population update. The immune memory particle Pg from the memory bank is replaced by the particle with poor adaptation value in the particle population Popk, forming a new generation particle population Popk +1 .

Step 6: When the preset maximum number of iterations or minimum error accuracy is reached, stop the iteration and output the optimal solution Pg, otherwise go to Step 2.

\section{Traffic flow prediction model based on immune particle swarm BP neural network}

\subsection{Determination of traffic flow data}

In an urban traffic network, the traffic conditions on a road section at a given time are closely related to the traffic conditions on that road section and upstream and downstream road sections in the immediate period. Therefore, forecasts are based on the traffic flow on the upstream sections of the roadway during the previous periods that are highly correlated with the forecast period. In addition, the regularity of traffic demand is also reflected in the weekly cycle of travel demand. The traffic inflow at the west inlet of road section a (i.e. between junctions $\mathrm{AB}$ ) at time $\mathrm{t}, \mathrm{Ia}(\mathrm{t})$, is influenced by the north to east left turn flow at junction $\mathrm{A}, \mathrm{Oa}-1(\mathrm{t})$, the west to east straight flow, Oa-2(t), and the south to east right turn flow, Oa-3(t). The model sets the measurement and prediction period $\Delta t=3 \mathrm{~min}$, with $\mathrm{Ia}(\mathrm{t}), \mathrm{Ia}(\mathrm{t}-\Delta t), \mathrm{Ia}(\mathrm{t}-2-\Delta t)$, Oa-1 $(\mathrm{t})$, Oa-1(t$\Delta t)$, Oa-2(t), Oa-2(t- $\Delta t)$, Oa-3(t), Oa-3(t- $\Delta t)$ and the traffic inflow of the day before road section a, Ia(t-24h) and the traffic inflow of the week before road section a The 11 observations, including $\mathrm{Ia}(\mathrm{t}-7 \times 24 \mathrm{~h})$, are used as historical data for predicting the traffic inflow $\mathrm{Ia}(\mathrm{t}+\Delta t)$ at road section a at time $\mathrm{t}+\Delta t$. 


\subsection{Pre-processing of traffic flow data}

Based on the transfer functions between the input, hidden and output layers, the input vectors are pre-processed to initialise the mapping and transform them into continuous output quantities in the corresponding interval, enabling an arbitrary non-linear mapping from input to output. The model is a three-layer BP neural network with only one hidden layer, and the transfer functions $f$ between the input layer, hidden layer and output layer are chosen as tangent function tansig and linear function purelin respectively, and the output range of the whole neural network is [-1,1]. Each input vector is converted to a value between $[-1,1]$ according to equation (7), so that the input vectors can be distributed more symmetrically in the non-saturated area of the tansig function to improve the approximation of the input-output mapping relationship of the BP neural network.

$$
x^{\prime}=-1+\frac{2 \times\left(x-x_{\min }\right)}{x_{\max }-x_{\min }}
$$

where $\mathrm{x}$ is the original input vector, $\mathrm{x}$ ' is the pre-processed input vector, $\mathrm{xmax}$ and xmin denote the maximum and minimum values of $\mathrm{x}$.

\subsection{Determining the parameter range of a neural network}

\subsubsection{Determine the initialization range for the number of hidden layer nodes, initial weights, and thresholds}

The number of input vectors in the three-layer BP neural network is Input num $=11$ and the number of output vectors is Output num $=1$. The number of nodes in the hidden layer, Mid num, is determined in the range $[5,14]$ according to equation

$$
\text { Mid num }=\sqrt{\text { Input num }+ \text { Output num }}+\alpha .
$$

where $\alpha$ is $1-10$. Since the range of the input vector after pre-processing is $[-1,1]$, the absolute value of the sum of the initial weights of each node should be less than 1 to ensure that each neuron can perform the initial operation at the position where their activation function changes the most, therefore, the range interval of both the initial weights and the threshold value is $[-1,1]$.

\subsubsection{Setting the particle fitness function and particle concentration function}

In order to find the particle that minimizes the error of the neural network, the model sets the individual fitness function Fitness of the particle according to equation

$$
\text { Fitness }(\cdot)=1 /\left(T_{i}-O_{i}\right)^{2}
$$

where $\mathrm{Ti}$ is the ideal output of particle $\mathrm{i}$, Oi is the actual output of particle $\mathrm{i}$. The concentration function $\mathrm{D}$ and the concentration selection probability $\mathrm{P}$ of the immune particles are defined according to equations (5) and (6). 


\subsection{Immune particle swarm algorithm to train the neu- ral network and determine the initial structure of the network}

According to 4.3, the range of parameters of the neural network structure is determined and the training samples are input. The immune particle swarm algorithm is used to train neural networks with different numbers of hidden layer nodes one by one until the maximum number of evolutionary generations or the minimum error accuracy is achieved. The optimal neural network structure is determined by comparison, and the optimal number of hidden layer nodes, initial weights and threshold values are obtained.

\subsection{Training neural networks using the BP algorithm}

Matlab algorithm commands are used to train the optimal neural network model, iterate until the specified accuracy is reached, and finally output the initial weights, thresholds, actual training times and errors of the BP neural network: NET=train(net,P,T). NET is the neural network created by the immune particle swarm algorithm, and NET is the neural network trained by the $\mathrm{BP}$ algorithm. $\mathrm{p}$ and $\mathrm{t}$ represent the input and output vectors of the training samples respectively.

\subsection{Simulation output and data reduction}

The simulation output of the traffic flow using BP neural network, Matlab algorithm command is: $\mathrm{Y}=\operatorname{sim}(\mathrm{NET}, \mathrm{P} 1)$. Where $\mathrm{P} 1$ is the input vector, $\mathrm{Y}$ is the simulation output of the input vector P1. Finally, the simulation output Y is reduced by referring to equation (7) to obtain the predicted traffic flow.

\section{Traffic Forecasting Experiments and Analysis of Results}

The traffic flow of Yangqiao Middle Road (2nd Ring Road towards Baima Road) in Fuzhou City was used as the traffic prediction section. The traffic flow at Yangqiao Road intersection of the 2nd Ring Road was counted from 9:30-10:30 for two consecutive weeks, and 50 sets of traffic data with a time interval of 3 min from 9:30-10:15 were randomly selected from the record set as training

samples for the The neural network was trained. The maximum number of training generations was 1000 , and the minimum error was set to 0.000001 .

\subsection{Comparison of simulation output results}

Five sets of traffic flow data from 10:15-10:30 on 11 November 2015 were extracted, and the neural networks were trained with Immune Particle Swarm 
IMPSO, Basic Particle Swarm PSO and Fast BP algorithms for simulation output respectively, and the following results were obtained:

$I M P S O A N N=0.0524 \quad-0.1721 \quad 0.6949 \quad 0.255 \quad 1 \quad 0.188 \quad 5$

$P S O A N N=0.1758-0.2195 \quad 0.8829 \quad 0.0981 \quad 0.1037 \quad 0.1716-0.0615$

The actual measured traffic flow data are as follows:

$$
\mathrm{Y}=0.0400 \quad-0.2000 \quad 0.6000 \quad 0.2800 \quad 0.1200
$$

The simulation output was reduced according to equation (7), where $x \max =51$ and $x \min =26$. The traffic flow predictions are shown in Table. The compar-

\begin{tabular}{cccccc}
\hline Time & $10: 18$ & $10: 21$ & $10: 24$ & $10: 27$ & $10: 30$ \\
\hline Actual & 39 & 36 & 46 & 42 & 40 \\
IMPSOANN & 39.1551 & 36.3489 & 47.1863 & 41.6883 & 40.8558 \\
PSOANN & 40.6977 & 35.7559 & 48.5368 & 38.7257 & 37.2039 \\
BPANN & 35.9500 & 33.0538 & 35.2888 & 40.6450 & 37.7313 \\
\hline
\end{tabular}

ison between the simulation output and the actual flow can be seen that the simulated flow obtained by the immune particle swarm algorithm is a better fit to the actual flow than the neural network trained by the basic particle swarm algorithm and the fast BP algorithm.

\section{Conclusion}

The immune particle swarm algorithm can perform global search based on the population intelligence formed by the information communication among the population particles, which avoids the shortcomings of the particle swarm algorithm in balancing the population convergence and individual diversity. The structure of the neural network trained by the immune particle swarm algorithm can overcome the inherent shortcomings of the neural network such as slow convergence and easy to fall into local optimum. The experimental results show that the BP neural network trained by the immune particle swarm algorithm can effectively improve the prediction accuracy of traffic flow and reduce the prediction error.

\section{References}

[1] O. Kotavaara, H. Antikainen, and J. Rusanen, "Urbanization and transportation in finland, 1880-1970," Journal of Interdisciplinary History, vol. 42, no. 1, pp. 89-109, 2011.

[2] Y. Li, L. Jia, W. Wu, J. Yan, and Y. Liu, "Urbanization for rural sustainability-rethinking china's urbanization strategy," Journal of Cleaner Production, vol. 178, pp. 580-586, 2018.

[3] M. B. Arvin, R. P. Pradhan, and N. R. Norman, "Transportation intensity, urbanization, economic growth, and co2 emissions in the g-20 countries," Utilities Policy, vol. 35, pp. 50-66, 2015 .

[4] R. Arnott, A. De Palma, and R. Lindsey, "Does providing information to drivers reduce traffic congestion?" Transportation Research Part A: General, vol. 25, no. 5, pp. 309-318, 1991. 
[5] J. J. Q. Yu and J. Gu, "Real-time traffic speed estimation with graph convolutional generative autoencoder," IEEE Transactions on Intelligent Transportation Systems, vol. 20, no. 10, pp. 3940-3951, Oct. 2019.

[6] X. Zou, S. Zhang, C. Zhang, J. J. Q. Yu, and E. Chung, "Long-term origin-destination demand prediction with graph deep learning," IEEE Transactions on Big Data, 2021, in press.

[7] K. K. Dewan and I. Ahmad, "Carpooling: a step to reduce congestion," Engineering Letters, vol. 14, no. 1, pp. 61-66, 2007.

[8] J. J. Q. Yu, "Travel mode identification with GPS trajectories using wavelet transform and deep learning," IEEE Transactions on Intelligent Transportation Systems, 2020, in press.

[9] C. Pasquale, I. Papamichail, C. Roncoli, S. Sacone, S. Siri, and M. Papageorgiou, "Two-class freeway traffic regulation to reduce congestion and emissions via nonlinear optimal control," Transportation Research Part C: Emerging Technologies, vol. 55, pp. 85-99, 2015.

[10] J. J. Q. Yu, "Sybil attack identification for crowdsourced navigation: a self-supervised deep learning approach," IEEE Transactions on Intelligent Transportation Systems, 2020, in press.

[11] J. J. Q. Yu and A. Y. S. Lam, "Autonomous vehicle logistic system: Joint routing and charging strategy," IEEE Transactions on Intelligent Transportation Systems, vol. 19, no. 7, pp. 21752187, Jul. 2018.

[12] H.-F. Yang, T. S. Dillon, and Y.-P. P. Chen, "Optimized structure of the traffic flow forecasting model with a deep learning approach," IEEE transactions on neural networks and learning systems, vol. 28, no. 10, pp. 2371-2381, 2016.

[13] J. J. Q. Yu, Y. Hou, A. Y. S. Lam, and V. O. K. Li, "Intelligent fault detection scheme for microgrids with wavelet-based deep neural networks," IEEE Transactions on Smart Grid, vol. 10, no. 2, pp. 1694-1703, Mar. 2019.

[14] X. Jiang and H. Adeli, "Dynamic wavelet neural network model for traffic flow forecasting," Journal of transportation engineering, vol. 131, no. 10, pp. 771-779, 2005.

[15] J. J. Q. Yu, Y. Hou, and V. O. K. Li, "Online false data injection attack detection with wavelet transform and deep neural networks," IEEE Transactions on Industrial Informatics, vol. 14, no. 7, pp. 3271-3280, Jul. 2018.

[16] Y. Lv, Y. Duan, W. Kang, Z. Li, and F.-Y. Wang, "Traffic flow prediction with big data: a deep learning approach," IEEE Transactions on Intelligent Transportation Systems, vol. 16, no. 2, pp. 865-873, 2014.

[17] B. Hamner, "Predicting travel times with context-dependent random forests by modeling local and aggregate traffic flow," in 2010 IEEE International Conference on Data Mining Workshops. IEEE, 2010, pp. 1357-1359.

[18] J. J. Q. Yu, C. Markos, and S. Zhang, "Long-term urban traffic speed prediction with deep learning on graphs," IEEE Transactions on Intelligent Transportation Systems, 2021, in press.

[19] C. Zhang, S. Zhang, J. J. Q. Yu, and S. Yu, "FASTGNN: A topological information protected federated learning approach for traffic speed forecasting," IEEE Transactions on Industrial Informatics, 2021, in press.

[20] J. J. Q. Yu, "Citywide traffic speed prediction: a geometric deep learning approach," Knowledge-Based Systems, p. 106592, Nov. 2020.

[21] __ , "Semi-supervised deep ensemble learning for travel mode identification," Transportation Research Part C: Emerging Technologies, vol. 112, pp. 120-135, Mar. 2020.

[22] C. P. Green, J. S. Heywood, and M. N. Paniagua, "Did the london congestion charge reduce pollution?" Regional Science and Urban Economics, vol. 84, p. 103573, 2020.

[23] E. Castillo, J. M. Menéndez, and S. Sánchez-Cambronero, "Predicting traffic flow using bayesian networks," Transportation Research Part B: Methodological, vol. 42, no. 5, pp. 482$509,2008$. 
[24] J. J. Q. Yu, W. Yu, and J. Gu, "Online vehicle routing with neural combinatorial optimization and deep reinforcement learning," IEEE Transactions on Intelligent Transportation Systems, vol. 20, no. 10, pp. 3806-3817, Oct. 2019.

[25] N. G. Polson and V. O. Sokolov, "Deep learning for short-term traffic flow prediction," Transportation Research Part C: Emerging Technologies, vol. 79, pp. 1-17, 2017.

[26] J. Dong and H. S. Mahmassani, "Stochastic modeling of traffic flow breakdown phenomenon: Application to predicting travel time reliability," IEEE Transactions on Intelligent Transportation Systems, vol. 13, no. 4, pp. 1803-1809, 2012.

[27] S. Sun, C. Zhang, and G. Yu, "A bayesian network approach to traffic flow forecasting," IEEE Transactions on intelligent transportation systems, vol. 7, no. 1, pp. 124-132, 2006. 